\title{
Exploring the anti-proliferative activity of Pelargonium sidoides DC with in silico target identification and network pharmacology
}

\author{
ASP Pereira ${ }^{1}$, MJ Bester and Z Apostolides ${ }^{*}, 1$ \\ ${ }^{1}$ Department of Biochemistry, Faculty of Natural and Agricultural Sciences, University of Pretoria, Pretoria, South \\ Africa, ${ }^{2}$ Department of Anatomy, Faculty of Health Sciences, University of Pretoria, Pretoria, South Africa.
}

*Address correspondence to this author at the Department of Biochemistry, University of Pretoria, Hillcrest 0083, Pretoria, South Africa; Tel: +27-12-420-2486; Fax:+27-12-362-5302; Email: Zeno.Apostolides@up.ac.za

\begin{abstract}
Pelargonium sidoides DC (Geraniacea) is a medicinal plant indigenous to Southern Africa that has been widely evaluated for its use in the treatment of upper respiratory tract infections. In recent studies, the anti-proliferative potential of $P$. sidoides was shown and several phenolic compounds were identified as the bioactive compounds. Little, however, is known regarding their anti-proliferative protein targets. In this study, the anti-proliferative mechanisms of $P$. sidoides through in silico target identification and network pharmacology methodologies were evaluated. The protein targets of the 12 phenolic compounds were identified using the target-identification server PharmMapper and the server for predicting Drug Repositioning and Adverse Reactions via the Chemical-Protein Interactome (DRAR$\mathrm{CPI}$. Protein-protein and protein-pathway interaction networks were subsequently constructed with Cytoscape 3.4.0 to evaluate potential mechanisms of action. A total of 142 potential human target proteins were identified with the in silico target identification servers and 90 of these were found to be related to cancer. The protein interaction network was constructed from 86 proteins involved in 209 interactions with each other, and two protein clusters were observed. A pathway enrichment analysis identified over 80 Kyoto Encyclopedia of Genes and Genomes (KEGG) pathways enriched with the protein targets and included several pathways specifically related to cancer as well as various signaling pathways that have been found to be dysregulated in cancer. These results indicate that the anti-proliferative activity of $P$. sidoides may be multifactorial and arises from the collective regulation of several interconnected cell signaling pathways.
\end{abstract}

Keywords: Pelargonium sidoides, anti-proliferative, target identification, network pharmacology, signaling pathways, kinase, PharmMapper, Cytoscape 


\section{Introduction}

Pelargonium sidoides DC (Geraniacea) is a medicinal plant indigenous to South Africa and Lesotho that has traditionally been used for the treatment of tuberculosis as well as various stomach ailments, such as colic, diarrhea and dysentery [1]. It has, however, in more recent times, been developed into medicinal formulations like EPs® 7630 and Linctagon for the treatment of upper respiratory tract infections [1-5]. Laboratory-based studies have revealed that the beneficial effects of extracts prepared from the roots of $P$. sidoides can be attributed to their antibacterial, antiviral and immunomodulatory bioactivities [6-15]. Extracts have been shown to be rich in a variety of phenolic compounds such as gallic acid, highly oxygenated simple coumarins as well as proanthocyanidins [16].

In previous studies, we found anti-proliferative activity for a commercial radix mother tincture of $P$. sidoides in vitro and through a bioactivity-guided approach; we isolated seven fractions with significant anti-proliferative activity $[17,18]$. Liquid chromatography with tandem mass spectrometry detection (LC-MS/MS) analysis of these fractions revealed the presence of phenolic compounds gallic acid, koaburaside, proanthocyanidin dimers as well as a trimer, dihydroxycoumarin sulfates, trihydroxycoumarin as well as three turgorins namely leaf movement factor (LMF) 2, LMF3 and the periodic leaf movement factor 1 (PLMF1). A new phenolic acid glycoside sulfate (PGS) not previously shown in $P$. sidoides extracts was identified according to its mass fragmentation pattern. Although anti-proliferative activity has been shown for gallic acid and proanthocyanidins, it remains largely unknown how these compounds downregulate the expression of drug targets, such as cyclin-dependent kinases (CDK) 2 and 4, Bcl- 2, nuclear factor kappa-light-chain-enhancer of activated B cells (NF$\mathrm{KB})$, extracellular regulated kinase (ERK) $1 / 2$ as well as matrix metalloproteinase (MMP) 2 and 9 [19-22]. Furthermore, little is known regarding the potential anti-proliferative activity of the turgorins, phenolic glycosides and the coumarin sulfates and how these compounds may contribute to the observed anticancer activity of the fractions.

In silico target identification coupled with network pharmacological profiling has been gaining momentum and played an important role in the development of new computational methods to predict drug-target networks as well as drug discovery, drug re-purposing and drug-target validation in medicinal chemistry and pharmacology [23-30]. In the study of medicinal plants and their bioactive compounds, in silico target identification coupled with network pharmacological profiling has provided valuable insights into potential disease models, protein targets as well as mechanisms of action [31-35]. In the current study, we have implemented these methodologies in an attempt to identify the potential protein targets of the isolated compounds to provide insights into the anti-proliferative activity of $P$. sidoides. 


\section{Materials and methods}

\section{In silico target fishing}

The two-dimensional structures of gallic acid, koaburaside, 6,7-dihydroxycoumarin-8-sulfate (67DiOHC8S), 7,8-dihydroxycouamrin-6-sulfate (78DiOHC6S), 6,7,8-trihydroxycoumarin (678TriOHC), gallocatechin-gallocatechin dimer (GCGC), gallocatechin-gallocatechingallocatechin trimer (GCGCGC), catechin-gallocatechin (CGC), LMF2, LMF3, PLMF1 and PGS were created with Advanced Chemistry Development (ACD)/ChemSketch freeware version 12.02, 2010 [36] and the smiles notation representing each of the structures was generated. From the smiles notation, the 3D structure was generated and optimized with University of California, San Francisco (UCSF) Chimera version 1.11 (build 41097) [37,38] and saved as mol2 files. These mol2 files were subsequently uploaded to the PharmMapper and DRAR-CPI servers for in silico screening [39,40]. Following the screening, potential protein targets were identified using a cutoff fit-value of 4.000 together with a positive zscore for PharmMapper while for DRAR-CPI, a cutoff docking score of -50.000 with a zscore of -1.500 was used. Only human protein targets were considered. Literature sources, as well as the Disease and Gene Database [41,42], were consulted to determine whether the potential protein targets identified were related to cancer. The compound-target-cancer network was generated by Cytoscape version 3.4.0 [43], and the NetworkAnalyzer Application version 2.7 [44] was used to evaluate some basic network features.

\section{Protein interaction network and protein-pathway enrichment analysis}

The gene symbols of the protein targets identified were submitted to the String Database $[45,46]$ to evaluate the potential protein interactions amongst the targets. Only protein interactions with the highest confidence score (0.900) were considered. The protein interaction network was constructed with Cytoscape version 3.4.0 following the removal of all isolated nodes. To determine where the protein targets may play a role, pathway enrichment analysis of the KEGG pathways was performed with the String Database. Only the enriched pathways with a False Discovery Rate (FDR) of 0.001 or less were considered in the construction of the protein-pathway network with Cytoscape version 3.4.0.

\section{Results and discussion}

\section{In silico target fishing}

The 12 compounds identified from the bioactive fractions isolated from $P$. sidoides were screened in silico with PharmMapper and DRAR-CPI to elucidate the potential antiproliferative protein targets of these compounds. Screening with the PharmMapper server 
involves docking the compounds with pharmacophore models of the active site of protein targets while with DRAR-CPI, the compounds are docked into the protein pockets of the protein target $[39,40]$.

The PharmMapper server revealed 53 different human potential protein targets for the compounds using a cutoff fit-value of 4.000 and a positive z-score value [35]. Some of the compounds were found to have high fit-values for different pharmacophore models representing the same protein target. This was observed for 67DiOHC8S that showed high fit-values for four pharmacophore models of tyrosine-protein phosphatase non-receptor type 1 (PTPN1) and 78DiOHC6S for three pharmacophore models of CDK2. Furthermore, some of the protein targets identified were found to be potential targets of more than one compound. This was observed for protein targets actin, alpha skeletal muscle 1 (ACTA1), cystathionine beta-synthase (CBS), cystic fibrosis transmembrane conductance regulator (CFTR), HRas, membrane metallo-endopeptidase (MME), 6-phosphofructo-2-kinase (PFKFB1), PTPN1, uridine-cytidine kinase 2 (UCK2).

The DRAR-CPI server revealed another 97 different potential human protein targets with a cutoff docking score of -50.000 and z-score values smaller than -1.5. As observed with the PharmMapper server, some of the protein targets identified were found to be potential targets of more than one compound. This was observed for 35 different protein targets. Some protein targets were also shared between the two servers as seen with 67DiOHC8S and target CBS, PGS and glutathione S-transferase A1 (GSTA1) as well as target PTPN1 and potential inhibitors PLMF1 and 67DiOHC8S. Taken collectively, 142 different human proteins were found to be potential targets of the 12 compounds (Fig. 1). PLMF1 and PGS were found to be the least selective with 49 and 47 potential targets, respectively. Both have a sulfated sugar moiety that may play an important role in forming intermolecular hydrogen bonds with the active site of the target proteins. On the other hand, the GCGCGC trimer, gallic acid and 678 TriOHC were found to be most selective with three, five and four potential targets, respectively. The GCGCGC trimer has a very large and bulky structure and this likely limits its access to the protein's active site while for gallic acid and 678TriOHC, their relatively small structures with only a few hydrogen bond donors and acceptors likely limits their interactions with the protein's active site. It was observed that similar structures shared similar potential protein targets, and this could be seen from PLMF1 and PGS that shared 20 protein targets, LMF2 and LMF3 shared five, the two dihydroxycoumarin sulfates shared eight while the proanthocyanidin dimers shared four. Of the 142 potential human target proteins that were identified with the in silico target fishing servers, 90 were found to be related to cancer, based on literature sources as well as the Disease and Gene Database 
(Fig. 1). These in silico results provide support for the observed anti-proliferative activity of $P$. sidoides with in vitro studies.

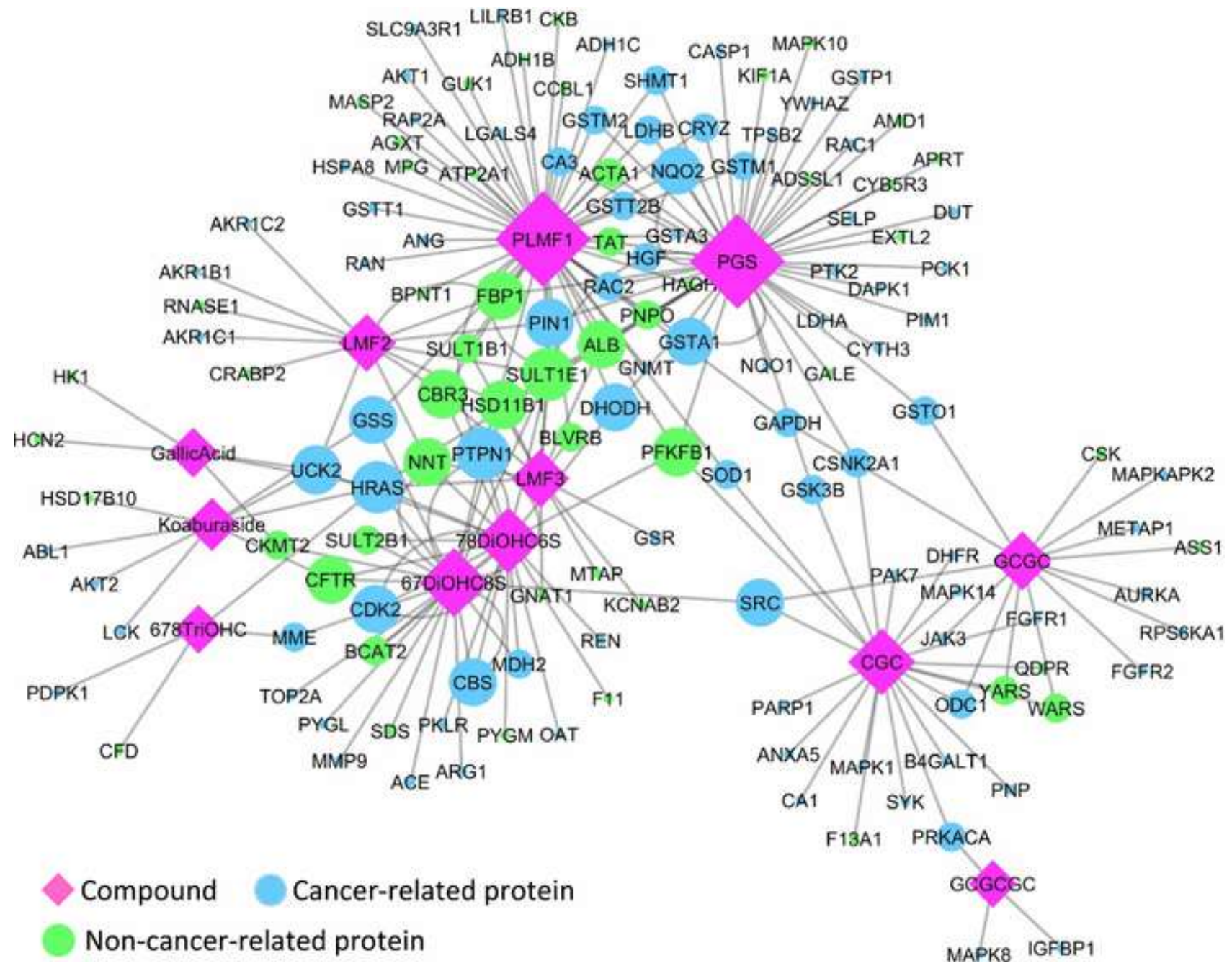

Fig. 1. Compound-target-cancer network. The network was generated with Cytoscape version 3.4 .0 with the NetworkAnalyzer Application version 2.7. All protein targets are represented by their gene symbol. Size of compound markers reflects the number of targets for each compound, while size of protein marker reflects number of compounds that are possible inhibitors. Loops within the network indicate that the compound was a possible inhibitor for different models of the same protein target

\section{Protein interaction network and pathway enrichment analysis}

In order to explore where these protein targets may play a role, a protein interaction network as well as pathway enrichment analysis were performed [35]. The protein interaction network in Fig. 2 was constructed with the String Database using the highest confidence score $(0.900)$ and excluding all isolated nodes. Within this protein interaction network, two large clusters connected by glutathione S-transferase P (GSTP1) were observed. These clusters suggest that the bioactive compounds of $P$. sidoides are targeting proteins that are either part of the same protein complex or involved in the same pathway [47]. 


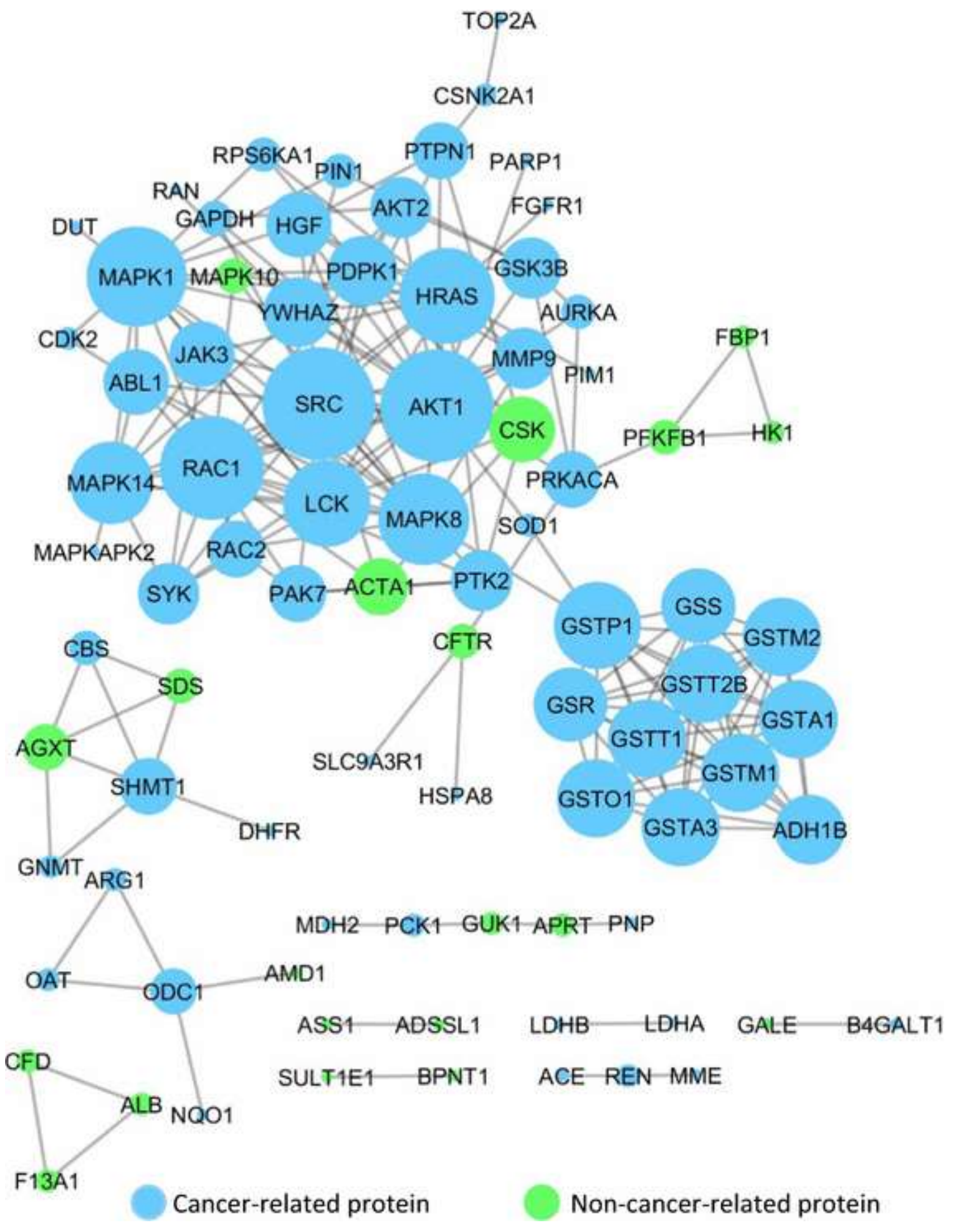

Fig. 2. Protein-protein interaction network. Protein-protein interactions were evaluated with the String Database at confidence level of 0.900 . Following the removal of all isolated nodes, the protein-protein interaction network was generated with Cytoscape version 3.4.0 with the NetworkAnalyzer Application version 2.7. Size of protein marker reflects number of interactions with other proteins

With the pathway enrichment analysis, over 80 KEGG pathways with FDR values of less than 0.001 were found to be enriched with the protein targets. Fourteen pathways relating specifically to cancer were identified and included pathways in cancer, proteoglycans in 
cancer, chemical carcinogenesis, colorectal cancer, prostate cancer, pancreatic cancer, renal cell carcinoma, endometrial cancer, viral carcinogenesis, melanoma, non-small cell lung cancer, acute myeloid leukemia, chronic myeloid leukemia and glioma (Table 1). Several signaling pathways were also identified and some of these pathways have been found to be dysregulated in cancer as has been observed with the ErbB, vascular endothelial growth factor (VEGF), mitogen-activated protein kinase (MAPK), Ras, phosphatidylinositol-3-kinase (PI3K)-Akt, Rap1, 5'-adenosine monophosphate-activated protein kinase (AMPK), Wnt, focal adhesion, hypoxia inducible factor (HIF-1) and mammalian target of rapamycin (mTOR) signaling pathways [48-58]. Dysregulation of these pathways has been found to be associated with increased cell proliferation, cell survival, inhibition of apoptosis, cell migration and invasion as well as angiogenesis.

Table 1. Enriched KEGG pathways related to cancer

\begin{tabular}{llll}
\hline Pathway ID & Pathway description & Proteins & FDR \\
\hline \multicolumn{2}{l}{ Cancer-related pathways } & \multicolumn{3}{l}{} \\
hsa05200 & Pathways in cancer & 15 & $1.24 \mathrm{e}-10$ \\
hsa05205 & Proteoglycans in cancer & 13 & $1.52 \mathrm{e}-10$ \\
hsa05204 & Chemical carcinogenesis & 9 & $2.71 \mathrm{e}-10$ \\
hsa05210 & Colorectal cancer & 8 & $1.56 \mathrm{e}-09$ \\
hsa05215 & Prostate cancer & 8 & $2.86 \mathrm{e}-08$ \\
hsa05212 & Pancreatic cancer & 7 & $6.29 \mathrm{e}-08$ \\
hsa05211 & Renal cell carcinoma & 7 & $8.31 \mathrm{e}-08$ \\
hsa05213 & Endometrial cancer & 6 & $4.79 \mathrm{e}-07$ \\
hsa05203 & Viral carcinogenesis & 9 & $5.13 \mathrm{e}-07$ \\
hsa05218 & Melanoma & 6 & $2.74 \mathrm{e}-06$ \\
hsa05223 & Non-small cell lung cancer & 5 & $1.52 \mathrm{e}-05$ \\
hsa05221 & Acute myeloid leukemia & 5 & $1.62 \mathrm{e}-05$ \\
hsa05220 & Chronic myeloid leukemia & 5 & $4.62 \mathrm{e}-05$ \\
hsa05214 & Glioma & 4 & 0.000466 \\
Cancer-related signaling pathways & & \\
hsa04012 & ErbB signaling pathway & 11 & $2.55 \mathrm{e}-12$ \\
hsa04370 & VEGF signaling pathway & 10 & $2.55 \mathrm{e}-12$ \\
hsa04510 & Focal adhesion & 14 & $5.31 \mathrm{e}-12$ \\
hsa04010 & MAPK signaling pathway & 14 & $7.24 \mathrm{e}-11$ \\
hsa04014 & Ras signaling pathway & 13 & $1.52 \mathrm{e}-10$ \\
hsa04151 & PI3K-Akt signaling pathway & 14 & $1.87 \mathrm{e}-09$ \\
hsa04915 & Estrogen signaling pathway & 8 & $5.27 \mathrm{e}-08$ \\
hsa04015 & Rap1 signaling pathway & 10 & $1.24 \mathrm{e}-07$ \\
hsa04152 & AMPK signaling pathway & 7 & $3.83 \mathrm{e}-06$ \\
hsa04310 & Wnt signaling pathway & $8.32 \mathrm{e}-06$ \\
hsa04066 & HIF-1 signaling pathway & $2.17 \mathrm{e}-05$ \\
hsa04150 & mTOR signaling pathway & $2.19 \mathrm{e}-05$ \\
\hline
\end{tabular}




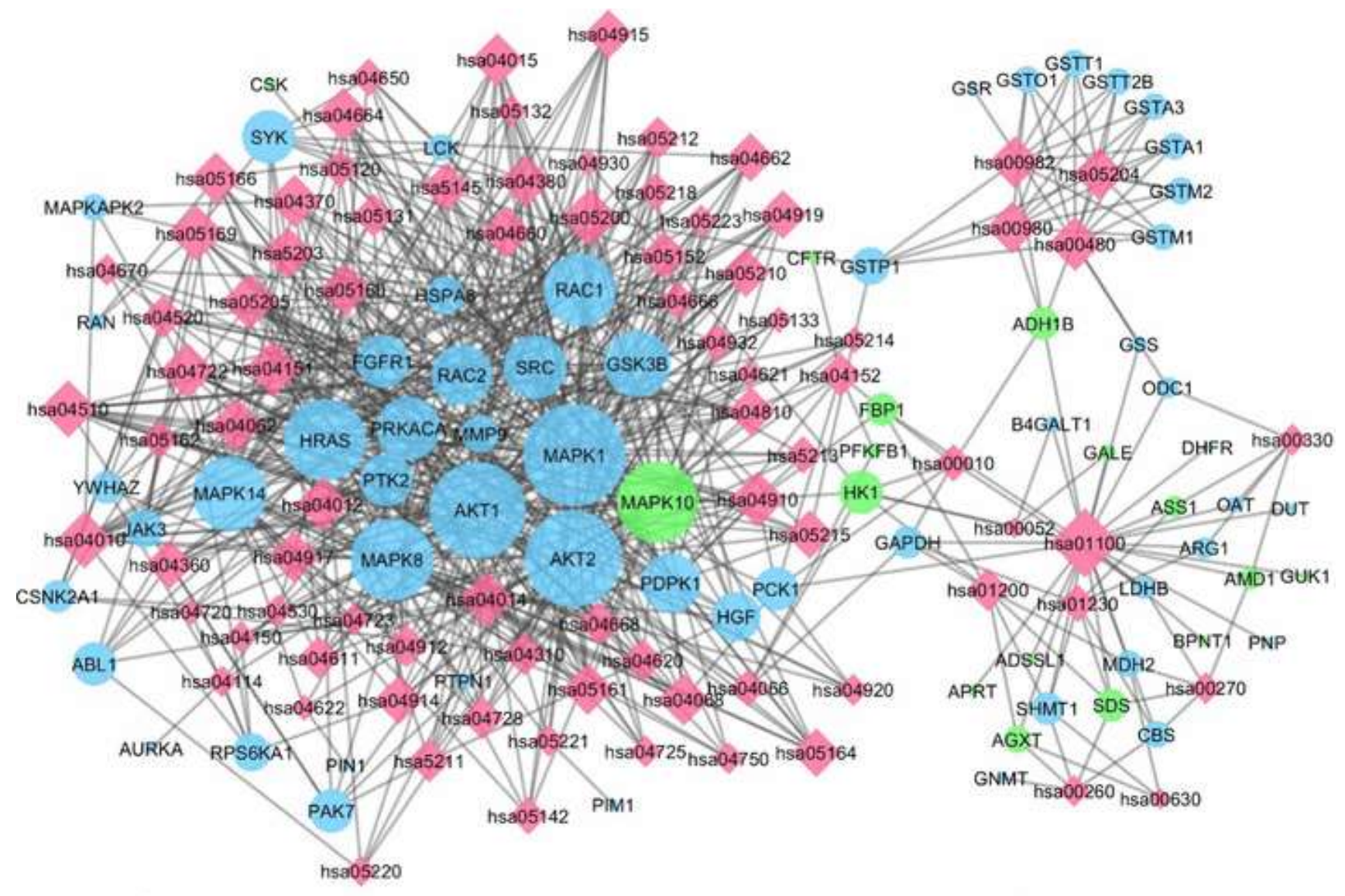

Cancer-related protein

Non-cancer-related protein

Pathway ID

Fig. 3. Protein-pathway interaction network. Pathway enrichment analysis of the KEGG pathways was evaluated with the String Database. The protein-pathway interaction network was constructed by Cytoscape version 3.4.0 with the NetworkAnalyzer Application version 2.7. Only pathways that were found to be enriched with a FDR of 0.001 were included. Pathways are represented by their KEGG pathway ID. Size of the protein markers reflects the number of pathways that are associated with, while the size of the pathway marker reflects the number of the protein targets that are involved in that specific pathway

Inspection of the protein targets involved in these pathways revealed that some of these targets are involved in multiple pathways suggesting that these pathways are interrelated. This can be seen in Fig. 3 where a group of about 25 proteins namely phosphoenolpyruvate carboxykinase (PCK1), hepatocyte growth factor (HGF), Pak7, fibroblast growth factor receptor (FGFR), Rac 1 and 2, focal adhesion kinase (FAK), 3-phosphoinositide-dependent protein kinase 1 (PDPK1), Akt 1 and 2, glycogen synthase kinase-3 beta (GSK3 $\beta$ ), Ras, Abl1, Src, cAMP-dependent protein kinase catalytic subunit alpha (PRKACA), MMP9, Heat shock cognate 71 kiloDalton protein (HSPA8), mitogen-activated protein kinase-activated protein kinase 2 (MAPKAPK2), Lck, Syk, casein kinase II subunit alpha (CSNK2A1) as well as the MAPKs $1,8,10$ and 14 that were all found to be involved in the majority of the pathways identified. Several of these protein targets are concerned with protein kinase activity (GO0004672) involving the phosphorylation of amino acid residues in proteins 
(G00006468) suggesting that phenolic compounds as those identified in $P$. sidoides may specifically target the protein kinases (serine/threonine and tyrosine kinases) involved in signal transduction.

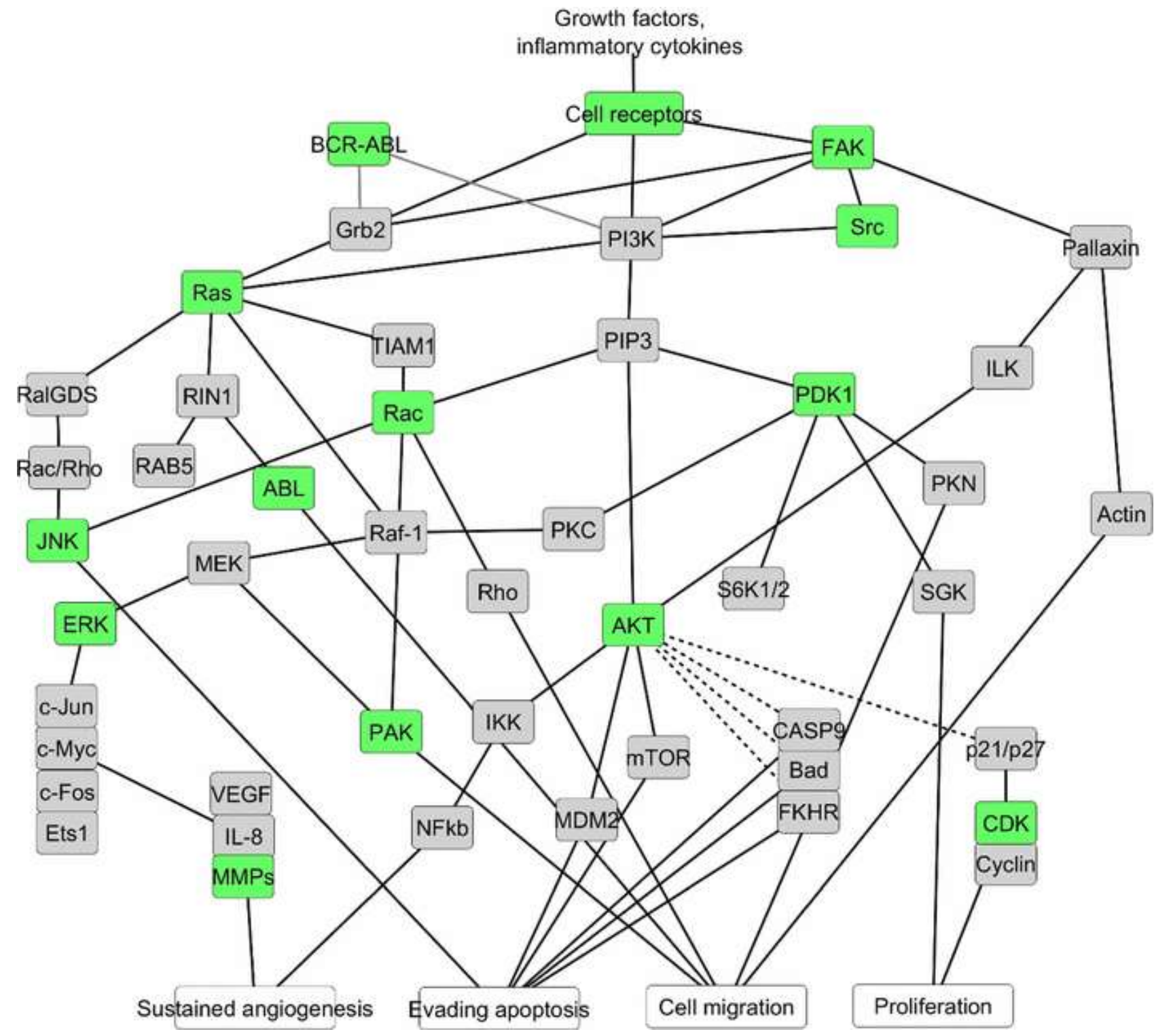

Fig. 4. Potential regulation of PI3K-Akt and Ras/MAPK pathways by the phenolic compounds of $P$. sidoides. Pathways were adapted from hsa05200 Pathways in cancer from the KEGG Database. Protein targets highlighted in green represent the potential targets of the $P$. sidoides compounds

The interplay between these pathways in cancer pathogenesis can be seen in Fig. 4 and the protein targets of the bioactive compounds of $P$. sidoides have been highlighted. Regulation of these pathways by the bioactive compounds of $P$. sidoides should result in the experimentally observed cell growth inhibition, cell cycle arrest and induction of apoptosis. There is evidence in the literature supporting the observed results here. Phenolic compounds similar to those that were identified in $P$. sidoides like gallic acid, 
proanthocyanidins and hydroxylated simple coumarins have shown, following treatment of cancer cells in vitro, altered expression of PI3K, Akt, Ras, Rac1, MMP9, NF-KB, FAK, CDK2, MAPK-ERK and c-Jun N-terminal kinase (JNK) resulting in cell growth inhibition, cell cycle arrest and apoptosis [19,22,59-61].

The cell signaling pathways identified here are also under oxidative control as has been observed with the PI3K-Akt and the Janus Kinase (JAK)- signal transducers and activators of transcription protein (STAT) signaling pathways as well as the Src protein kinases that can then go on to activate the PI3K-Akt, Ras/Raf/MAPK and STAT pathways [62-67]. The bioactive compounds identified from $P$. sidoides were also shown to have significant antioxidant activity in vitro [17]. Thus, $P$. sidoides besides regulating these signal transduction pathways through possible direct interaction with the concerned protein kinases, may also regulate these same pathways by scavenging the reactive oxygen and nitrogen species that can also activate these pathways.

The glutathione pathway (hsa00480) was also found to be enriched with an FDR of 1.36e08, and it was not surprising that some of the compounds were found to be potential substrates of the GSTs. The GSTs are enzymes that conjugate glutathione to xenobiotics for detoxification [68]. Several anticancer drugs have been found to be substrates of the GSTs, and the GSTs have been implicated in chemotherapeutic drug resistance development. The $P$. sidoides compounds may thus through their interaction with the GSTs, deplete glutathione and reduce the excretion of these drugs thus prolonging their therapeutic effects. This depletion may be beneficial for drugs that have a short half-life but harmful if a drug has a long half-life as toxicity may then become a factor with the longer exposure times. Besides their role in detoxification pathways, the GSTs have been found to be upregulated in several cancer types and have been found to play a role in the regulation of the MAP kinase pathway by inhibiting JNK-induced apoptosis [68]. The $P$. sidoides compounds thus by targeting the GSTs may result in an anti-proliferative effect by preventing the GSTs from inhibiting JNK and allowing the cells to undergo stress-induced apoptosis. This speculation suggests that a combination therapy of $P$. sidoides extract with anti-cancer drugs may be particularly effective, by draining away glutathione, thus prolonging the half-life of the drugs, and inhibiting GSTs so that JNK-induced apoptosis of the cancer cells may proceed unhindered.

Also of particular interest, was that these pathways are activated by the inflammatory mediators, cytokines and growth factors, and extracts of $P$. sidoides has previously shown anti-inflammatory activity in vivo [14]. Besides inflammation, the protein targets and activation of these signaling pathways are also found to play important roles during innate 
immune responses to generate a defense to invading pathogens. $P$. sidoides extracts have also been found to exert immunomodulatory activity $[6,9,10]$. The enriched pathways concerned with the innate immune responses include Fc epsilon RI signaling pathway, leukocyte transendothelial migration, chemokine signaling pathway, Toll-like receptor signaling pathways, natural killer cell mediated cytotoxicity, Fc gamma R-mediated phagocytosis, nucleotide-binding oligomerization domain (NOD)-like receptor signaling pathway and retinoic acid-inducible gene 1 (RIG-I)-like receptor signaling pathway (Table 2). The results presented here may thus also suggest the inflammatory pathways regulated by $P$. sidoides extracts as well as provide evidence for the immunomodulation associated with $P$. sidoides extracts. Also, the immune system has been shown to play an important role in cancer progression and drugs such as thalidomide and pomalidomide that can inhibit the action of some of the inflammatory mediators and enhance the activity of immune effectors have proven useful in the treatment of cancer [69-71]. Thus, the immunomodulatory activity of $P$. sidoides may also account for its anti-proliferative activity.

Table 2. Enriched KEGG pathways related to immune function and infection

\begin{tabular}{|c|c|c|c|}
\hline Pathway ID & Pathway description & Proteins & FDR \\
\hline \multicolumn{4}{|c|}{ Immune-related pathways } \\
\hline hsa04664 & Fc epsilon RI signaling pathway & 11 & $3.18 \mathrm{E}-13$ \\
\hline hsa04062 & Chemokine signaling pathway & 11 & 3.16E-09 \\
\hline hsa04660 & T cell receptor signaling pathway & 9 & 4.24E-09 \\
\hline hsa04662 & $\mathrm{B}$ cell receptor signaling pathway & 8 & 6.19E-09 \\
\hline hsa04620 & Toll-like receptor signaling pathway & 7 & $1.55 \mathrm{E}-06$ \\
\hline hsa04668 & TNF signaling pathway & 7 & $2.01 \mathrm{E}-06$ \\
\hline hsa04666 & Fc gamma R-mediated phagocytosis & 6 & 8.96E-06 \\
\hline hsa04650 & Natural killer cell mediated cytotoxicity & 6 & $5.68 \mathrm{E}-05$ \\
\hline hsa04621 & NOD-like receptor signaling pathway & 4 & 0.000342 \\
\hline hsa04670 & Leukocyte transendothelial migration & 5 & 0.000428 \\
\hline hsa04622 & RIG-I-like receptor signaling pathway & 4 & 0.000707 \\
\hline \multicolumn{4}{|c|}{ Pathways related to infection } \\
\hline hsa05169 & Epstein-Barr virus infection & 13 & 3.63E-11 \\
\hline hsa05160 & Hepatitis C & 9 & $3.60 \mathrm{E}-08$ \\
\hline hsa05131 & Shigellosis & 7 & 4.14E-08 \\
\hline hsa05161 & Hepatitis B & 9 & $6.50 \mathrm{E}-08$ \\
\hline hsa05145 & Toxoplasmosis & 8 & $1.91 \mathrm{E}-07$ \\
\hline hsa05120 & Epithelial cell signaling in Helicobacter pylori infection & 6 & 2.01E-06 \\
\hline hsa05164 & Influenza A & 8 & $2.88 \mathrm{E}-06$ \\
\hline hsa05152 & Tuberculosis & 8 & 3.38E-06 \\
\hline hsa05166 & HTLV-I infection & 9 & 5.57E-06 \\
\hline hsa05142 & Chagas disease (American trypanosomiasis) & 6 & $1.52 \mathrm{E}-05$ \\
\hline hsa05162 & Measles & 6 & 7.35E-05 \\
\hline hsa05132 & Salmonella infection & 5 & $9.68 \mathrm{E}-05$ \\
\hline hsa05133 & Pertussis & 4 & 0.000707 \\
\hline
\end{tabular}


The results of this study highlight not only the potential polypharmacological nature of the $P$. sidoides compounds evaluated here but also the complexity of treating a disease like cancer. Cancer is a complex multi-step disease involving mutations and genomic instability leading to uncontrolled cell growth, resistance to cell death, angiogenesis, invasion, inflammation and immune evasion [72]. Over the years, drug discovery has moved from the idea of a "single drug single target" to a "single drug multiple targets" idea [73-75]. Although the polypharmacological nature of drug compounds may be viewed as an undesirable feature due to the possibility of adverse reactions, looking at the complexity of cancer as a disease this "single drug multiple targets" idea or "multiple drugs multiple targets" may be better suited to its therapeutic treatment. This is observed with the use of multi-kinase inhibitors like sunitinib, axitinib, lapatinib and sorafenib that target several protein kinases as well as combination therapy in cancer treatment strategies [74].

The majority of the compounds evaluated here were predicted to have more than 10 potential protein targets, with the exception being gallic acid, the trihydroxycoumarin as well as the bulky GCGCGC proanthocyanin trimer. The main type of protein targets of these compounds were the protein kinases that were found to be involved in Ras-MAPK and PI3KAKT signaling pathways (Fig. 4). The P. sidoides compounds can potentially not only affect the activity of several upstream regulators of these two pathways Ras, Src, FAK, FGFR and BCR-ABL but also several of the downstream effectors of these two pathways. This is noteworthy because some of these downstream effectors can be activated by other proteins such as Protein Kinase C, JAK, cAMP and CDC42 (not shown, see KEGG pathways hsa04151 and hsa04010). This will thus lead to not only the attenuation of the Ras-MAPK and PI3K-AKT signaling pathways but also possibly the attenuation of other signaling pathways. Also, there is much cross-talk between the Ras-MAPK and PI3K-AKT signaling pathways and studies have shown the down regulation of the one pathway results in an upregulation of the other, thus the downregulation of both pathways may be needed for effective treatment strategies [76].

The importance of the potential polyphamacological nature of the $P$. sidoides compounds is particularly evident when looking as the tumor microenvironment. The Ras-MAPK and PI3KAKT signaling pathways play not only a role in the proliferation and growth of the tumor cells but also the surrounding cells in the tumor environment like the endothelial cells, fibroblasts and cells of the immune system [77]. As an example, activation of these pathways by the binding of growth factors or cytokines to their respective receptor on the cell surface of the tumor cell will promote the proliferation and growth of the tumor cells [78]. The tumor cells also through the activation of these pathways will also result in the production and secretion of growth factors and cytokines that give important signals to the surrounding cells of the 
tumor microenvironment. Upon the release of VEGF by the tumor cells and their subsequent binding to the VEGFR on endothelial cells and pericytes, the Ras-MAPK and PI3K-AKT pathways will be activated to stimulate the proliferation and survival of these cells as well as their migration through the activation of FAK and MAPK 14 (also both targets for the $P$. sidoides compounds) $[57,79]$. Thus, the $P$. sidoides compounds besides inhibiting the growth of the tumor cells through regulation of the PI3K-AKT and Ras-MAPK pathways may also inhibit the process of angiogenesis through the attenuation of the PI3K-AKT and Ras-MAPK pathways in the cells of the vascular system. The VEGF signaling pathway (hsa04370) was found to be enriched for the protein targets of the $P$. sidoides compounds (Table 1).

Similarly, the release of growth factors from the tumor cells can act on the surrounding cells of the extracellular matrix (ECM) like the fibroblasts and through the activation of the PI3KAKT and Ras-MAPK pathways, promote their proliferation, survival, migration and subsequent ECM remodeling allowing for tumor migration [80]. Also, regulation of these two key pathways by the $P$. sidoides compounds should have an anti-inflammatory effect by inhibiting the release and production of inflammatory cytokines and growth factors from immune cells like the macrophages and neutrophils that are associated with the tumor microenvironment and drive tumorigenesis $[62,77]$. This is evident from the immune related pathways that were found to be enriched with the PI3K-AKT and Ras-MAPK protein targets of the $P$. sidoides compounds (Table 2).

Thus, through the collective regulation/inhibition of these protein targets by the bioactive compounds of $P$. sidoides that are involved in parallel and interlinked pathways and are important to several cell types found in the tumor microenvironment, a significant effect on not only tumor cell proliferation (as observed with our in vitro studies) but on the tumorigenesis process as a whole should be observed with in vivo studies. The $P$. sidoides compounds need not necessarily be strong binders of these protein targets but by potentially binding to and affecting the activity of multiple proteins and weakening their interactions with each other, the $P$. sidoides compounds should efficiently attenuate these signaling pathways resulting in reduced cancer cell proliferation, migration and evasion [73]. Also, by inhibiting several parallel signal transduction pathways, there may also be less of a chance of resistance development [74]. The polypharmacological nature of compounds and in particular phenolic compounds also suggest why these compounds show beneficial effects in the treatment of a wide variety of diseases. This is observed with green tea and its primary bioactive compounds the catechins that have been shown in previous studies to have beneficial effects in the prevention and treatment of diseases like cancer, Alzheimer's, cardiovascular diseases, diabetes and infections [35]. 


\section{Conclusions}

In this study, in silico target identification coupled with network pharmacology have provided important insights into the anti-proliferative activity of $P$. sidoides and further provided support for the bioactivity observed in vitro. The major protein targets identified were shown to be protein kinases involved in signal transduction events particularly those concerned with the Ras/MAPK and PI3K-Akt signaling pathways that have been found to be dysregulated in cancer. The results also provided insight into the anti-inflammatory and immunomodulatory activity that has been found in vivo to be associated with $P$. sidoides extracts. These results suggest that the anti-proliferative activity of $P$. sidoides may be multifactorial and arises from the collective regulation of several interconnected cell signaling pathways. It remains to be seen whether further in vitro and in vivo studies will reflect the in silico results presented here.

\section{Acknowledgements}

We would like to acknowledge the National Research Foundation of South Africa for funding this project.

\section{Declaration of interest}

All authors declare no conflict of interest.

\section{References}

1. Brendler T, Van Wyk B (2008) A historical, scientific and commercial perspective on the medicinal use of Pelargonium sidoides (Geraniaceae). J Ethnopharmacol 119: 420-433. doi:10.1016/j.jep.2008.07.037

2. Matthys H, Eisebitt R, Seith B, Heger M (2003) Efficacy and safety of an extract of Pelargonium sidoides (EPs 7630) in adults with acute bronchitis: A randomised, doubleblind, placebo-controlled trial. Phytomedicine 10: 7-17. doi:10.1078/1433-187X-00308

3. Matthys $\mathrm{H}$, Kamin W, Funk P, Heger M (2007) Pelargonium sidoides preparation (EPs ${ }^{\circledR}$ 7630) in the treatment of acute bronchitis in adults and children. Phytomedicine 14: 69-73. doi:10.1016/j.phymed.2006.11.015 
4. Chuchalin AG, Berman B, Lehmacher W (2005) Treatment of acute bronchitis in adults with a pelargonium sidoides preparation (EPs 7630): a randomized, double-blind, placebocontrolled trial. Explore (NY) 1: 437-445. doi:10.1016/j.explore.2005.08.009

5. Lizogub VG, Riley DS, Heger M (2007) Efficacy of a Pelargonium sidoides preparation in patients with the common cold: a randomized, double blind, placebo-controlled clinical trial. Explore (NY) 3: 573-584. doi:10.1016/j.explore.2007.09.004

6. Conrad A, Hansmann C, Engels I, Daschner FD, Frank U (2007) Extract of Pelargonium sidoides (EPs 7630) improves phagocytosis, oxidative burst, and intracellular killing of human peripheral blood phagocytes in vitro. Phytomedicine 14 (Suppl 6): 46-51. doi:10.1016/j.phymed.2006.11.016

7. Lewu F, Grierson D, Afolayan A (2006) The leaves of Pelargonium sidoides may substitute for its roots in the treatment of bacterial infections. Biol Conserv 128: 582-584. doi:10.1016/j.biocon.2005.10.018

8. Kayser O, Kolodziej H (1997) Antibacterial activity of extracts and constituents of Pelargonium sidoides and Pelargonium reniforme. Planta Med 63: 508-510. doi:10.1055/s2006-957752

9. Kayser O, Kolodziej H, Kiderlen A (2001) Immunomodulatory principles of Pelargonium sidoides. Phytother Res 15: 122-126. doi:10.1002/ptr.785

10. Kolodziej H, Kayser O, Radtke OA, Kiderlen AF, Koch E (2003) Pharmacological profile of extracts of Pelargonium sidoides and their constituents. Phytomedicine 10 (Suppl 4): 1824. doi:10.1078/1433-187X-00307

11. Koch E, Hauer H, Stumpf K (2006) Use of Pelargonium sidoides and Pelargonium reniforme root extracts. US Patent 2006-0263448.

12. Mativandlela S, Lall N, Meyer JJM (2006) Antibacterial, antifungal and antitubercular activity of (the roots of) Pelargonium reniforme (CURT) and Pelargonium sidoides (DC) (Geraniaceae) root extracts. S Afr J Bot 72: 232-237. doi:10.1016/j.sajb.2005.08.002

13. Michaelis M, Doerr HW, Cinatl J Jr (2011) Investigation of the influence of $\operatorname{EPs}(R) 7630$, a herbal drug preparation from Pelargonium sidoides, on replication of a broad panel of respiratory viruses. Phytomedicine 18: 384-386. doi:10.1016/j.phymed.2010.09.008 
14. Noldner M, Schotz K (2007) Inhibition of lipopolysaccharid-induced sickness behavior by a dry extract from the roots of Pelargonium sidoides (EPs 7630) in mice. Phytomedicine 14 (Suppl 6): 27-31. doi:10.1016/j.phymed.2006.11.013

15. Schnitzler P, Schneider S, Stintzing FC, Carle R, Reichling J (2008) Efficacy of an aqueous Pelargonium sidoides extract against herpesvirus. Phytomedicine 15: 1108-1116. doi:10.1016/j.phymed.2008.06.009

16. Kolodziej H (2007) Fascinating metabolic pools of Pelargonium sidoides and Pelargonium reniforme, traditional and phytomedicinal sources of the herbal medicine Umckaloabo®. Phytomedicine 14: 9-17. doi:10.1016/j.phymed.2006.11.021

17. Pereira A, Bester M, Soundy P, Apostolides Z (2015) Activity-guided isolation and identification of the major antioxidant and anticancer compounds from a commercial Pelargonium sidoides tincture. Med Chem Res 24: 3838-3852. doi:10.1007/s00044-015$1425-6$

18. Pereira A, Bester M, Soundy P, Apostolides Z (2016) Anti-proliferative properties of commercial Pelargonium sidoides tincture, with cell-cycle $\mathrm{G}_{0} / \mathrm{G}_{1}$ arrest and apoptosis in Jurkat leukaemia cells. Pharm Biol 54: 1831-1840. doi:10.3109/13880209.2015.1129545

19. Ho H, Chang C, Ho W, Liao SY, Wu CH, Wang CJ (2010) Anti-metastasis effects of gallic acid on gastric cancer cells involves inhibition of NF-KB activity and downregulation of PI3K/AKT/small GTPase signals. Food Chem Toxicol 48: 2508-2516.

doi:10.1016/j.fct.2010.06.024

20. Meeran SM, Katiyar SK (2007) Grape seed proanthocyanidins promote apoptosis in human epidermoid carcinoma A431 cells through alterations in Cdki-Cdk-cyclin cascade, and caspase- 3 activation via loss of mitochondrial membrane potential. Exp Dermatol 16: 405-415. doi:10.1111/j.1600-0625.2007.00542.x

21. Rajalingam K, Schreck R, Rapp UR, Albert Š (2007) Ras oncogenes and their downstream targets. Biochim Biophys Acta 1773: 1177-1195.

doi:10.1016/j.bbamcr.2007.01.012

22. Vayalil PK, Mittal A, Katiyar SK (2004) Proanthocyanidins from grape seeds inhibit expression of matrix metalloproteinases in human prostate carcinoma cells, which is associated with the inhibition of activation of MAPK and NF kappa B. Carcinogenesis 25: 987-995. doi:10.1093/carcin/bgh095 
23. Arodz T, Bonchev D, Diegelmann RF (2013) A network approach to wound healing. Adv Wound Care 2: 499-509. doi:10.1089/wound.2012.0386

24. Chandrasekaran S, Bonchev D (2013) A network view on Parkinson's disease. Comput Struct Biotechnol J 7: 1-18. doi:10.5936/csbj.201304004

25. Durán FJR, Alonso N, Caamaño O, García-Mera X, Yañez M, Prado-Prado FJ, González-Díaz H (2014) Prediction of multi-target networks of neuroprotective compounds with entropy indices and synthesis, assay, and theoretical study of new asymmetric 1, 2rasagiline carbamates. Int J Mol Sci 15: 17035-17064. doi:10.3390/ijms150917035

26. González-Díaz H, Herrera-Ibatá DM, Duardo-Sánchez A, Munteanu CR, OrbegozoMedina RA, Pazos A (2014) ANN multiscale model of anti-HIV drugs activity vs AIDS prevalence in the US at county level based on information indices of molecular graphs and social networks. J Chem Inf Model 54: 744-755. doi:10.1021/ci400716y

27. Prado-Prado F, García-Mera X, Escobar M, Sobarzo-Sánchez E, Yañez M, RieraFernandez P, González-Díaz H (2011) 2D MI-DRAGON: a new predictor for protein-ligands interactions and theoretic-experimental studies of US FDA drug-target network, oxoisoaporphine inhibitors for MAO-A and human parasite proteins. Eur J Med Chem 46: 5838-5851. doi:10.1016/j.ejmech.2011.09.045

28. Prado-Prado F, Garcia-Mera X, Escobar M, Alonso N, Caamano O, Yanez M, GonzalezDiaz H (2012) 3D MI-DRAGON: new model for the reconstruction of US FDA drug-target network and theoretical-experimental studies of inhibitors of rasagiline derivatives for AChE. Curr Top Med Chem 12: 1843-1865. doi:10.2174/156802612803989228

29. Romero-Durán FJ, Alonso N, Yañez M, Caamaño O, García-Mera X, González-Díaz H (2016) Brain-inspired cheminformatics of drug-target brain interactome, synthesis, and assay of TVP1022 derivatives. Neuropharmacol 103: 270-278.

doi:10.1016/j.neuropharm.2015.12.019

30. Thomas S, Bonchev D (2010) A survey of current software for network analysis in molecular biology. Hum Genomics 4: 353-360. doi:10.1186/1479-7364-4-5-353

31. Bhattacharjee B, Chatterjee J (2013) Identification of proapoptopic, anti-inflammatory, anti-proliferative, anti-invasive and anti-angiogenic targets of essential oils in cardamom by dual reverse virtual screening and binding pose analysis. Asian Pac J Cancer Prev 14: 37353742. doi:10.7314/APJCP.2013.14.6.3735 
32. Lei Q, Liu H, Peng Y, Xiao P (2015) In silico target fishing and pharmacological profiling for the isoquinoline alkaloids of Macleaya cordata (Bo Luo Hui). Chinese Medicine 10:37. doi:10.1186/s13020-015-0067-4

33. Ma X, Lv B, Li P, Jiang X, Zhou Q, Wang X, Gao X (2016) Identification of "multiple components-multiple targets-multiple pathways" associated with Naoxintong capsule in the treatment of heart diseases using UPLC/Q-TOF-MS and network pharmacology. Evid Based Complement Alternat Med 2016:1-15. doi:10.1155/2016/9468087

34. Shao Y, Qiao L, Wu L, Sun X, Zhu D, Yang, X, Mao X, Chen W, Liang W (2016) Structure Identification and Anti-Cancer Pharmacological Prediction of Triterpenes from Ganoderma lucidum. Molecules 21: 678. doi:10.3390/molecules21050678

35. Zhang S, Shan L, Li Q, Wang X, Li S, Zhang Y, Fu J, Liu X, Li H, Zhang W (2014) Systematic analysis of the multiple bioactivities of green tea through a network pharmacology approach. Evid Based Complement Alternat Med 2014: 512081. doi:10.1155/2014/512081

36. ACD/Chemsketch, version 12.02, Advanced Chemistry Development, Inc., Toronto, On, Canada, http://www.acdlabs.com.

37. UCSF Chimera, version 1.11, Resource for Biocomputing, Visualization, and Informatics at the University of California, San Francisco (supported by NIGMS P41-GM103311), http://www.rbvi.ucsf.edu/chimera

38. Pettersen EF, Goddard TD, Huang CC, Couch GS, Greenblatt DM, Meng EC, Ferrin TE (2004) UCSF Chimera--a visualization system for exploratory research and analysis. J Comput Chem 25 (13): 1605-1612. doi:10.1002/jcc.20084.

39. Liu X, Ouyang S, Yu B, Liu Y, Huang K, Gong J, Zheng S, Li Z, Li H, Jiang H (2010) PharmMapper server: a web server for potential drug target identification using pharmacophore mapping approach. Nucleic Acids Res 38: W609-W614. doi:10.1093/nar/gkq300

40. Luo H, Chen J, Shi L, Mikailov M, Zhu H, Wang K, He L, Yang L (2011) DRAR-CPI: a server for identifying drug repositioning potential and adverse drug reactions via the chemical-protein interactome. Nucleic Acids Res 39: W492-W498. doi:10.1093/nar/gkr299

41. Disease and Gene Annotations, Northwestern University Biomedical Informatics Center, NorthWestern University, Chicago, http://dga.nubic.northwestern.edu. 
42. Peng K, Xu W, Zheng J, Huang K, Wang H, Tong J, Lin Z, Liu J, Cheng W, Fu D, Du P, Kibbe WA, Lin SM, Xia T (2013) The Disease and Gene Annotations (DGA): an annotation resource for human disease. Nucleic Acids Res 41: D553-D560. doi:10.1093/nar/gks1244.

43. Cytoscape, version 3.4.0, Cytoscape Consortium, San Diego, http://www.cytoscape.org.

44. NetworkAnalyzer Application, version 2.7, Max-Planck-Institut für Informatik, Saarbrücken, http://med.bioinf.mpi-inf.mpg.de/netanalyzer/index.php.

45. String Database, version 10.0, String Consortium 2017, https://string-db.org.

46. Szklarczyk D, Franceschini A, Wyder S, Forslund K, Heller D, Huerta-Cepas J, Simonovic M, Roth A, Santos A, Tsafou KP, Kuhn M, Bork P, Jensen LJ, von Mering C (2015) STRING v10: protein-protein interaction networks, integrated over the tree of life. Nucleic Acids Res 43: D447-D452. doi:10.1093/nar/gku1003

47. Merico D, Gfeller D, Bader GD (2009) How to visually interpret biological data using networks. Nat Biotechnol 27: 921-924. doi:10.1038/nbt.1567

48. Bailey CL, Kelly P, Casey PJ (2009) Activation of Rap1 promotes prostate cancer metastasis. Cancer Res 69: 4962-4968. doi: 10.1158/0008-5472.CAN-08-4269

49. Dhillon AS, Hagan S, Rath O, Kolch W (2007) MAP kinase signalling pathways in cancer. Oncogene 26: 3279-3290. doi:10.1038/sj.onc.1210421

50. Downward J. (2003). Targeting RAS signalling pathways in cancer therapy. Nat Rev Cancer 3: 11-22. doi:10.1038/nrc969

51. Giles FJ (2001) The vascular endothelial growth factor (VEGF) signaling pathway: a therapeutic target in patients with hematologic malignancies. Oncologist 6 (Suppl 5): 32-39. doi:10.1634/theoncologist.6-suppl_5-32

52. Hynes NE, MacDonald G (2009) ErbB receptors and signaling pathways in cancer. Curr Opin Cell Biol 21: 177-184. doi:10.1016/j.ceb.2008.12.010

53. Jeon S, Hay N (2015) The double-edged sword of AMPK signaling in cancer and its therapeutic implications. Arch Pharm Res 38: 346-357. doi:10.1007/s12272-015-0549-z

54. Masoud GN, Li W (2015) HIF-1 a pathway: role, regulation and intervention for cancer therapy. Acta Pharm Sin B 5: 378-389. doi:10.1016/j.apsb.2015.05.007 
55. Polakis P (2012) Wnt signaling in cancer. Cold Spring Harb Perspect Biol 4: a008052. doi:10.1101/gad.14.15.1837

56. Pópulo H, Lopes JM, Soares $P$ (2012) The mTOR signalling pathway in human cancer. Int J Mol Sci 13: 1886-1918. doi:10.3390/ijms13021886

57. Tai Y, Chen L, Shen T (2015) Emerging roles of focal adhesion kinase in cancer. Biomed Res Intl 2015: 690690. doi:10.1155/2015/690690

58. Vara JÁF, Casado E, de Castro J, Cejas P, Belda-Iniesta C, González-Barón M (2004) PI3K/Akt signalling pathway and cancer. Cancer Treat Rev 30: 193-204.

doi:10.1016/j.ctrv.2003.07.007

59. Lee SH, Park C, Jin C, Kim G, Moon S, Hyun JW, Lee WH, Choi BT, Kwon TK, Yoo YH (2008) Involvement of extracellular signal-related kinase signaling in esculetin induced G1 arrest of human leukemia U937 cells. Biomed Pharmacother 62: 723-729. doi:10.1016/j.biopha.2007.12.001

60. Velasco-Velázquez MA, Agramonte-Hevia J, Barrera D, Jiménez-Orozco A, GarcíaMondragón MJ, Mendoza-Patiño N, Landa A, Mandoki J (2003) 4-Hydroxycoumarin disorganizes the actin cytoskeleton in B16-F10 melanoma cells but not in B82 fibroblasts, decreasing their adhesion to extracellular matrix proteins and motility. Cancer Lett 198: 179186. doi:10.1016/S0304-3835(03)00333-1

61. Wang C, Hsieh Y, Chu C, Lin Y, Tseng T (2002) Inhibition of cell cycle progression in human leukemia HL-60 cells by esculetin. Cancer Lett 183: 163-168. doi:10.1016/S03043835(02)00031-9

62. Giannoni E, Buricchi F, Raugei G, Ramponi G, Chiarugi P (2005) Intracellular reactive oxygen species activate Src tyrosine kinase during cell adhesion and anchorage-dependent cell growth. Mol Cell Biol 25: 6391-6403. doi:10.1128/MCB.25.15.6391-6403.2005

63. Kwon J, Lee SR, Yang KS, Ahn Y, Kim YJ, Stadtman ER, Rhee SG (2004) Reversible oxidation and inactivation of the tumor suppressor PTEN in cells stimulated with peptide growth factors. Proc Natl Acad Sci USA 101: 16419-16424. doi:10.1073/pnas.0407396101

64. Lim S, Clément M (2007) Phosphorylation of the survival kinase Akt by superoxide is dependent on an ascorbate-reversible oxidation of PTEN. Free Radic Biol Med 42: 11781192. doi:10.1016/j.freeradbiomed.2007.01.013 
65. Simon AR, Rai U, Fanburg BL, Cochran BH (1998) Activation of the JAK-STAT pathway by reactive oxygen species. Am J Physiol 275: C1640-C1652.

66. Wang X, McCullough KD, Franke TF, Holbrook NJ (2000) Epidermal growth factor receptor-dependent Akt activation by oxidative stress enhances cell survival. J Biol Chem 275: 14624-14631. doi:10.1074/jbc.275.19.14624

67. Zhang J, Xing D, Gao X (2008) Low-power laser irradiation activates Src tyrosine kinase through reactive oxygen species-mediated signaling pathway. J Cell Physiol 217: 518-528. doi:10.1002/jcp.21529

68. Townsend DM, Tew KD (2003) The role of glutathione-S-transferase in anti-cancer drug resistance. Oncogene 22: 7369-7375. doi:10.1038/sj.onc.1206940

69. Grivennikov SI, Greten FR, Karin M (2010) Immunity, inflammation, and cancer. Cell 140: 883-899. doi:10.1016/j.cell.2010.01.025

70. Lucia MS, Torkko KC (2004) Inflammation as a target for prostate cancer chemoprevention: pathological and laboratory rationale. J Urol 171: S30-S35. doi:10.1097/01.ju.0000108142.53241.47

71. Melchert M, List A (2007) The thalidomide saga. Int J Biochem Cell Biol 39: 1489-1499. doi:10.1016/j.biocel.2007.01.022

72. Hanahan D, Weinberg RA (2011) Hallmarks of cancer: the next generation. Cell 144: 646-674. doi:10.1016/j.cell.2011.02.01

73. Csermely P, Agoston V, Pongor S (2005) The efficiency of multi-target drugs: the network approach might help drug design. Trends Pharmacol Sci 26: 178-182. doi:10.1016/j.tips.2005.02.007

74. Peters J (2013) Polypharmacology-foe or friend? J Med Chem 56: 8955-8971. doi:10.1021/jm400856t

75. Reddy AS, Zhang S (2013) Polypharmacology: drug discovery for the future. Expert Rev Clin Pharm 6: 41-47. doi:10.1586/ecp.12.74

76. Saini KS, Loi S, de Azambuja E, Metzger-Filho O, Saini ML, Ignatiadis M, Dancey JE, Piccart-Gebhart MJ (2013) Targeting the PI3K/AKT/mTOR and Raf/MEK/ERK pathways in 
the treatment of breast cancer. Cancer Treat Rev 39: 935-946.

doi:10.1016/j.ctrv.2013.03.009

77. Balkwill FR, Capasso M, Hagemann T (2012) The tumor microenvironment at a glance. J Cell Sci 125: 5591-5596. doi:10.1242/jcs.116392

78. Meier F, Schittek B, Busch S, Garbe C, Smalley K, Satyamoorthy K, Li G, Herlyn M (2005) The RAS/RAF/MEK/ERK and PI3K/AKT signaling pathways present molecular targets for the effective treatment of advanced melanoma. Front Biosci 10: 2986-3001. doi: $10.2741 / 1755$

79. Jiang B, Liu L (2008) PI3K/PTEN signaling in tumorigenesis and angiogenesis. Biochim Biophys Acta - Proteins Proteomics 1784: 150-158. doi:10.1016/j.bbapap.2007.09.008

80. Larsen M, Artym VV, Green JA, Yamada KM (2006) The matrix reorganized: extracellular matrix remodeling and integrin signaling. Curr Opin Cell Biol 18: 463-471. doi:10.1016/j.ceb.2006.08.009 